\title{
Accidental awareness while under general anaesthesia
}

\author{
Lu Chang ${ }^{1,2}$, Quehua Luo ${ }^{2,3}$, Yunfei Chai ${ }^{4}$, Haihua Shu ${ }^{1,2, *}$ \\ ${ }^{1}$ Department of Anesthesiology, Xinhua Hospital Affiliated to Shanghai Jiaotong University School of Medicine, Shanghai, China; \\ ${ }^{2}$ Department of Anesthesiology, Guangdong Second Provincial General Hospital, Guangzhou, China; \\ ${ }^{3}$ The Second School of Clinical Medicine, Southern Medical University, Guangzhou, China; \\ ${ }^{4}$ Department of Anesthesiology, Cardiovascular Institute of Guangdong Province, Guangdong Provincial People's Hospital, \\ Guangdong Academy of Medical Sciences, Guangzhou, China.
}

\begin{abstract}
Summary Accidental awareness during general anaesthesia may cause many intraoperative discomforts and bring further moderate to severe long-term symptoms including flashbacks, nightmares, hyperarousal or post-traumatic stress disorder. The incidence of awareness varied from $0.017 \%$ to $4 \%$ among studies. The relatively reliable incidence of intraoperative awareness with postoperative recall is $0.02 \%$. The reason causing awareness was unclear. Insufficient anaesthetic dosing was thought as the principal cause. Even awareness was not comprehensively understood, some endeavors have been raised to prevent or reduce it, including $i$ ) Reducing the insufficient anaesthetic dosing induced by negligence; ii) Providing close clinical observation and clinical parameters from the monitor such as bispectral index or electroencephalogram, as well as isolated forearm technique and passive brain-computer interface may bring some effects sometimes. Because current studies still have some flaws, further trials with new detecting approach, superior methodology and underlying aetiology are needed to unfasten the possible factors causing awareness.
\end{abstract}

Keywords: Accidental awareness, general anaesthesia

Unintended or accidental awareness during general anaesthesia (AAGA) was regarded as a failure in the general anaesthesia process. It was difficult to define awareness or AAGA accurately since consciousness and anaesthesia were also difficult to define. Unintentional or accidental consciousness during general anaesthesia (GA), without emphasizing recall, has been referred to as awareness earlier, while AAGA, defined as explicit recall of GA and could be spontaneously reported by the patient or detected by direct questioning or promoting, has been mentioned more $(1,2)$. Although AAGA was relatively uncommon, it brought negative experiences including but not limited to intraoperative hearing voices or equipment noises, sensation of paralysis or pain, awareness of tracheal intubation and inability to breathe, etc. Furthermore, moderate to severe symptoms including flashbacks or nightmares, avoidance of situations relating

*Address correspondence to:

Dr. Haihua Shu, Department of Anesthesiology, Xinhua Hospital Affiliated to Shanghai Jiaotong University School of Medicine, 1665\# Kongjiang Road, Shanghai 200092, China.

E-mail: shuhaihua@hotmail.com to the experience, hyperarousal and post-traumatic stress disorder (PTSD) may suffer in 79\% of patients who experienced AAGA long-term (3). However, no studies have provided any strong evidence that awareness without recall has important negative consequences to date (2).

The incidence of awareness is difficult to be accurately determined. The identification of awareness in most researches based on explicit postoperative recall ranges, spontaneous patient reports or structured postoperative interviews, such as the Brice Questionaire (6-12). The relatively reliable incidence of intraoperative awareness with postoperative recall is $0.02 \%$, while the incidence without explicit recall may be much higher $(3,7,13,14)$ (Table 1). Variation among studies in the proportion of patients with a possible awareness event may be up to 200 -fold ( $4 \%$ vs. $0.017 \%$ ) (Table 1). It was unclear whether the differences in incidence resulted from disparities in patient population, sample size, time of investigation, anaesthetic technique, study design, clinical severity, method of identification or definition of awareness. It was found that different definition of awareness, for instance, explicit recall 
Table 1. The incidence of accidental awareness during general anaesthesia $(\mathbf{2 0 0 0 - 2 0 1 7 )}(4,5)$

\begin{tabular}{|c|c|c|c|c|c|}
\hline Study and year & $\begin{array}{l}\text { Sample } \\
\text { size, N }\end{array}$ & Incidence & Method of identification & Study design & $\begin{array}{l}\text { Explicit postoperative } \\
\text { recalls or not }\end{array}$ \\
\hline $\begin{array}{l}\text { Sandin et al, } \\
2000\end{array}$ & 11,785 & $18(0.15 \%)$ & $\begin{array}{l}\text { Modified Brice questionnaire: } \\
\text { PACU, } 1-3 \text { days and } 7-14 \text { days } \\
\text { postoperatively }\end{array}$ & $\begin{array}{l}\text { Observational } \\
\text { prospective case study }\end{array}$ & Explicit recalls \\
\hline $\begin{array}{l}\text { Myles et al, } \\
2000\end{array}$ & 11,811 & $12(0.11 \%)$ & $\begin{array}{l}\text { Question and answer survey } \\
\text { within } 24 \mathrm{~h} \text { of surgery }\end{array}$ & $\begin{array}{l}\text { Observational } \\
\text { prospective case study }\end{array}$ & Explicit recalls \\
\hline $\begin{array}{l}\text { Myles et al, } \\
2004(11)\end{array}$ & $\begin{array}{l}\text { Total: } 2,463 \\
\text { BIS: } 1,225 \\
\text { Routine care: } \\
1,238\end{array}$ & $\begin{array}{l}\text { Total: } 13(0.52 \%) \\
\text { BIS: } 2(0.16 \%) \\
\text { Routine care: } \\
11(0.89 \%)\end{array}$ & $\begin{array}{l}\text { Modified Brice questionnaire: } \\
2-6 \mathrm{~h}, 24-36 \mathrm{~h} \text {, and } 30 \text { days } \\
\text { postoperatively }\end{array}$ & $\begin{array}{l}\text { Randomised } \\
\text { controlled trial }\end{array}$ & Explicit recalls \\
\hline $\begin{array}{l}\text { Andrade et al, } \\
2011\end{array}$ & $\begin{array}{l}\text { Total: } 5,731 \\
\text { BIS: } 2,861 \\
\text { ETAC: } 2,852\end{array}$ & $\begin{array}{l}\text { Total: } 9(0.15 \%) \\
\text { BIS: } 7(0.24 \%) \\
\text { ETAC: } 2(0.07 \%)\end{array}$ & $\begin{array}{l}\text { Modified Brice questionnaire: } \\
72 \mathrm{~h} \text { and } 30 \text { days postoperatively }\end{array}$ & $\begin{array}{l}\text { Randomised } \\
\text { controlled trial }\end{array}$ & Explicit recalls \\
\hline $\begin{array}{l}\text { Andrade et al, } \\
2011\end{array}$ & $\begin{array}{l}\text { Total: } 18,832 \\
\text { BIS: } 9,460 \\
\text { ETAC: } 9,376\end{array}$ & $\begin{array}{l}\text { Total: } 20(0.11 \%) \\
\text { BIS: } 8(0.08 \%) \\
\text { ETAC: } 11(0.12 \%)\end{array}$ & $\begin{array}{l}\text { Brice questionnaire: } 28-30 \text { days } \\
\text { postoperatively }\end{array}$ & $\begin{array}{l}\text { Randomised } \\
\text { controlled trial }\end{array}$ & Explicit recalls \\
\hline $\begin{array}{l}\text { Pandit et al, } \\
2014\end{array}$ & $2,766,600$ & $471(0.017 \%)$ & $\begin{array}{l}\text { Spontaneous complaints/reports } \\
\text { of awareness }\end{array}$ & $\begin{array}{l}\text { Cross-sectional } \\
\text { observational study }\end{array}$ & Explicit recalls \\
\hline $\begin{array}{l}\text { Andrade et al, } \\
2008\end{array}$ & 184 & $2(1.1 \%)$ & $\begin{array}{l}\text { IFT and postoperative structured } \\
\text { interview }\end{array}$ & $\begin{array}{l}\text { Observational } \\
\text { prospective case study }\end{array}$ & $\begin{array}{l}\text { No cases of explicit } \\
\text { postoperative recall }\end{array}$ \\
\hline $\begin{array}{l}\text { Sanders et al, } \\
2017\end{array}$ & 260 & $12(4 \%)$ & $\begin{array}{l}\text { IFT followed by modified Brice } \\
\text { questionnaire }\end{array}$ & $\begin{array}{l}\text { Observational } \\
\text { prospective case study }\end{array}$ & $\begin{array}{l}\text { No cases of explicit } \\
\text { postoperative recall }\end{array}$ \\
\hline Total & $2,817,666$ & $557(0.020 \%)$ & & & \\
\hline
\end{tabular}

IFT, isolated forearm technique; BIS, bispectral index; ETAC, end-tidal anaesthetic gas concentration.

was regard as necessary or not may differentiate the results mostly $(15,16)$. However, what was certain was that with upwards of millions admissions leading to surgical intervention annually, patients suffered from the accidental awareness could be an enormous number of cases.

What incentives awareness was not clear totally. Insufficient anaesthetic dosing, sometimes caused by administration equipment failure or the negligence of an anesthetist, was thought as the pivotal cause of awareness. However, some occult factors might cause consciousness and memory despite clinicians may consider adequate anaesthesia. It is unclear whether the reduced potency of anaesthetic was induced by a genetic contribution (17-20).

Even awareness was not comprehensively understood, some endeavors to prevent awareness have been advocated. Reducing the insufficient anaesthetic dosing induced by negligence should be the first step. Anaesthesia equipment before each use, especially vaporiser, circuit and drug-infusion pump, must be checked carefully. Drug error should be avoided by double-checking and labelling all drug syringes. Clinical parameters from the monitor or clinical signs being directly observed might work sometimes. The isolated forearm technique (IFT) was thought as the current gold standard for connected consciousness monitoring and was used to remind the anesthetist (21). Electroencephalogram derived bispectral index (BIS) or electroencephalogram (EEG) was once proved to be critical in preventing intraoperative awareness with explicit recall compared with clinical signs in most clinical researches $(8-10,12)$. However, a study has shown that the BIS protocol was less useful in preventing awareness than end-tidal anesthetic-agent concentration (9). Moreover, innovative techniques, such as passive brain-computer interface (BCI) based on an intention of movement may provide a foundation that would allow to detect awareness (22).

Although substantial progress has been made in understanding awareness about the incidence, consequences, and prevention, lack of gold standard of the definition, detection and prevention still prevent us from minimizing it. Thus, further trials about AAGA, especially with new detecting approach, superior methodology, underlying aetiology and novel results compared with the existing literature are still precious in the future.

\section{References}

1. Pandit JJ, Andrade J, Bogod DG, Hitchman JM, Jonker 
WR, Lucas N, Mackay JH, Nimmo AF, O'Connor K, O'Sullivan EP, Paul RG, Palmer JH, Plaat F, Radcliffe JJ, Sury MR, Torevell HE, Wang M, Hainsworth J, Cook TM. The 5th National Audit Project (NAP5) on accidental awareness during general anaesthesia: Summary of main findings and risk factors. Anaesthesia. 2014; 69:10891101.

2. Absalom AR, Green D. NAP5: The tip of the iceberg, or all we need to know? Br J Anaesth. 2014; 113:527-530.

3. Nightingale P. Accidental awareness under general anaesthesia in the United Kingdom and Ireland. J R Coll Physicians Edinb. 2014; 44:289-290.

4. Tasbihgou SR, Vogels MF, Absalom AR. Accidental awareness during general anaesthesia - a narrative review. Anaesthesia. 2018; 73:112-122.

5. Messina AG, Wang M, Vezina DP, Pace NL. Adjudication of awareness events. Br J Anaesth. 2018; 121:329-330.

6. Gan TJ, Habib AS, Miller TE, White W, Apfelbaum JL. Incidence, patient satisfaction, and perceptions of postsurgical pain: Results from a US national survey. Curr Med Res Opin. 2014; 30:149-160.

7. Sandin RH, Enlund G, Samuelsson P, Lennmarken C. Awareness during anaesthesia: A prospective case study. Lancet. 2000; 355:707-711.

8. Avidan MS, Zhang L, Burnside BA, Finkel KJ, Searleman AC, Selvidge JA, Saager L, Turner MS, Rao S, Bottros M, Hantler C, Jacobsohn E, Evers AS. Anesthesia awareness and the bispectral index. N Engl J Med. 2008; 358:10971108.

9. Avidan MS, Jacobsohn E, Glick D, Burnside BA, Zhang L, Villafranca A, Karl L, Kamal S, Torres B, O'Connor M, Evers AS, Gradwohl S, Lin N, Palanca BJ, Mashour GA; BAG-RECALL Research Group. Prevention of intraoperative awareness in a high-risk surgical population. N Engl J Med. 2011; 365:591-600.

10. Mashour GA, Shanks A, Tremper KK, Kheterpal S, Turner CR, Ramachandran SK, Picton P, Schueller C, Morris M, Vandervest JC, Lin N, Avidan MS. Prevention of intraoperative awareness with explicit recall in an unselected surgical population: A randomized comparative effectiveness trial. Anesthesiology. 2012; 117:717-725.

11. Myles PS, Leslie K, McNeil J, Forbes A, Chan MT. Bispectral index monitoring to prevent awareness during anaesthesia: The B-Aware randomised controlled trial. Lancet. 2004; 363:1757-1763.

12. Zhang C, Xu L, Ma YQ, Sun YX, Li YH, Zhang L, Feng CS, Luo B, Zhao ZL, Guo JR, Jin YJ, Wu G, Yuan W, Yuan ZG, Yue Y. Bispectral index monitoring prevent awareness during total intravenous anesthesia: A prospective, randomized, double-blinded, multi-center controlled trial. Chin Med J (Engl). 2011; 124:3664-3669.

13. Sanders RD, Gaskell A, Raz A, Winders J, Stevanovic A, Rossaint R, Boncyk C, Defresne A, Tran G, Tasbihgou S, Meier S, Vlisides PE, Fardous H, Hess A, Bauer RM, Absalom A, Mashour GA, Bonhomme V, Coburn $\mathrm{M}$, Sleigh J. Incidence of connected consciousness after tracheal intubation: A prospective, international, multicenter cohort study of the isolated forearm technique. Anesthesiology. 2017; 126:214-222.

14. Leslie K, Culwick MD, Reynolds H, Hannam JA, Merry AF. Awareness during general anaesthesia in the first 4,000 incidents reported to webAIRS. Anaesth Intensive Care. 2017; 45:441-447.

15. Pandit JJ. Isolated forearm - or isolated brain? Interpreting responses during anaesthesia - or 'dysanaesthesia'. Anaesthesia. 2013; 68:995-1000.

16. Pandit JJ. Acceptably aware during general anaesthesia: 'dysanaesthesia'--the uncoupling of perception from sensory inputs. Conscious Cogn. 2014; 27:194-212.

17. Ghoneim MM, Block RI, Haffarnan M, Mathews MJ. Awareness during anesthesia: Risk factors, causes and sequelae: A review of reported cases in the literature. Anesth Analg. 2009; 108:527-535.

18. Nickalls RW, Mahajan RP. Awareness and anaesthesia: Think dose, think data. Br J Anaesth. 2010; 104:1-2.

19. Cheng VY, Martin LJ, Elliott EM, Kim JH, Mount HT, Taverna FA, Roder JC, Macdonald JF, Bhambri A, Collinson N, Wafford KA, Orser BA. Alpha5GABAA receptors mediate the amnestic but not sedative-hypnotic effects of the general anesthetic etomidate. J Neurosci. 2006; 26:3713-3720.

20. Kretschmannova K, Hines RM, Revilla-Sanchez R, Terunuma M, Tretter V, Jurd R, Kelz MB, Moss SJ, Davies PA. Enhanced tonic inhibition influences the hypnotic and amnestic actions of the intravenous anesthetics etomidate and propofol. J Neurosci. 2013; 33:7264-7273.

21. Linassi F, Zanatta P, Tellaroli P, Ori C, Carron M. Isolated forearm technique: A meta-analysis of connected consciousness during different general anaesthesia regimens. Br J Anaesth. 2018; 121:198-209.

22. Rimbert S, Riff P, Gayraud N, Schmartz D, Bougrain L. Median nerve stimulation based BCI: A new approach to detect intraoperative awareness during general anesthesia. Front Neurosci. 2019; 13:622.

(Received August 16, Accepted August 30, 2019) 